\title{
The Influence of Exercise on Stress and Depression 運動對壓力及憂鐢的影響
}

\author{
Merav NAGEL \\ Cornerstone Counseling Center \\ Salt Lake City, USA \\ 馬䪵.尼爾 \\ 美國鹽湖城基石輔導中心
}

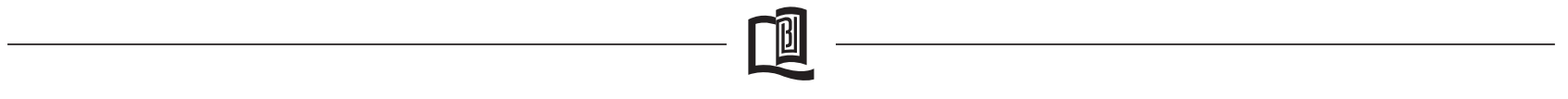

\begin{abstract}
There is evidence in human subjects that aerobic physical exercise boosts the immune system, influences stress hormone levels and depressive feelings (Brenner, Shek, Zamecnik, \& Shephard, 1998). Moderate physical exercise can prevent detrimental effects of stress and depression as a result of the interaction between the neuroendocrine system and the immune system post aerobic exercise (Fleshner, 2000). In this study, the contribution of intensity and duration of exercise to modulation of stress responses were assessed. Physical exercise modulation of the response to a stressful threatening event was examined in rats at the behavioral and biochemical levels. At the behavioral level, the following parameters were measured: anxiety (Elevated Plus Maze, Startle reflex response measures), depression (Learned helplessness) exploratory orientation (Open field) and of cognitive abilities (Spatial learning and memory, active avoidance). At the biochemical level, alterations in the basal concentrations of neurosteroids and in responsiveness to stress were measured.
\end{abstract}

In response to acute stress there is a rapid increase in neurosteroids (Vallee, Rivera, Koob, Purdy, \& Fitzgerald, 2000). The neurosteroid allopregnanolone has been shown to be a potent ligand of gamma-aminobutyric acid (GABA)-A receptors. It enhances its receptor-mediated inhibitory effects in the rat (Guo et al., 1995; Pericic, Svob, Jazvinscak, \& Mirkovic, 2000). The neurosteroid 3 alpha, 5 alpha THP acts like an antidepressant drug. It interacts with the central delta receptor, which has antidepressant-like effects (Khisti, Chopde, \& Jain, 2000; Reddy, Kaur, \& Kulkarni, 1998).

\section{摘 要}

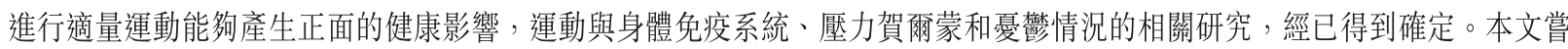
試以不同的運動強度及時期, 並用白鼠作用受試對象, 去探討運動與壓力兩者的生理變化。

\section{Research Objectives}

Brain researchers suggest that the ability to cope with stressful experiences improves with physical activity; however, little is known about the neural mechanisms and the critical parameters which define the effectiveness of physical exercise. Due to lack of empirical data, the objectives of the study were:
- To evaluate the hypothesis that physical exercise contributes to the ability to cope with a stress and depression.

- To verify the physiological basis of these effects and process using an animal model.

- To characterize the important variables which define the quality and intensity of these effects and process. 


\section{Background}

In the last decade several steroids were found to be present independently of peripheral steroidogenesis in the central nervous system. They were termed neurosteroids (Robel, Schumacher, \& Baulieu, 1999). Researchers report that neurosteroids were found to rapidly alter the excitability of $\mathrm{CNS}$ by bi-directional allosteric modulation of the GABA-A receptor $\mathrm{Cl}$ - ionophore complex (GRC) (Robel \& Baulieu, 1994). Neurosteroids affect anxiety-like behavior in response to stress and may be involved in depression (Reddy, et al., 1998). Preceding acute stress, there is an increase in neurosteroids concentration in the brain and plasma, as was documented in rats (Atsuo, Kaoru, Hiroshi, Yoshitake, \& Masao, 1995; Pericic et al., 2000). Large fluctuation of the neurosteroid 3 alpha hydroxy, 5 alpha pregnan 20 one (THP) are associated with changes in mood (Guidotti \& Costa, 1998). Under certain physiological conditions such as in stress and depression, THP, is one of the most potent endogenous modulators of GRC in the brain (Strohle at al., 1999). In mice, THP acts like an antidepressant to mice and causes reduction in immobility if they are facing learned helplessness situation (Khisti, et al., 2000). Drugs which operate the same way contain the same chemical compounds (e.g. metyrapone and fluoxetine) (Uzunov et al., 1998).

Neurosteroids are endogenous modulators of neural function responsible for a broad spectrum of physiological mechanisms (Vallee, et al., 2000). The body's response to physical or psychological stress includes the activation of the endocrine hypothalamic-pituitary adrenal (HPA) axis and the sympathetic nervous system (Atsuo et al., 1995). Intense aerobics exercise may be interpreted as a physical stress on the body because of large hormonal change (Atsuo et al., 1995). Under moderate aerobics exercise intensity (50-75\% of maximum heart rate) leukocyte concentration, which are mainly related to plasma norepinephrine concentration, increase (Brenner et al., 1998). Under high intensity (higher that $75 \%$ of maximum heart rate), plasma cortisol levels also rise (Brenner et al., 1998). High cortisol levels may maintain the lymphopenia and neutrocytosis (Pederson et al., 1997). High intensity exercise elicits the same mechanism causes by stress; elevation of cortisol level, blood lactate, and blood pressure (Kaciuba et al., 1994). As opposed to high intensity and short duration exercise, long duration aerobic exercise, (3-5 times per week), allows the body to revert to homeostasis, and have an improved and trained endocrine system (Fleshner, 2000).
Moderate aerobic exercise in contrast to high intensity and short duration exercise, lowers heart rate and systolic blood pressure baseline, and therefore is considered to promote performance of the cardiovascular and respiratory systems (Boutcher \& Nugent, 1993; Brownley, West, Hinderliter, \& Light, 1996; McCubbib et al., 1992; Spalding, Jeffers, Porges, \& Hatfield, 2000). Other types of exercise such as relaxation techniques: Yoga, Jacobson, diaphragmatic breathing, can also lower heart rate and systolic blood pressure. However, they do not have the same prolonged physiological cardiovascular benefits of aerobic exercise (Anshel, 1995). The steroid compound ouabainlike (OLC) has been identified as an active ingredient in controlling blood pressure and cardiorenal function during aerobic exercise (Atsuo et al., 1995). Aerobic exercise and relaxation techniques may contribute to emotional balance, mood regulation and an overall psychological well being, aerobic exercise is sometimes used as a therapeutic tool for depression (Fox, 1999; Perna, Antoni, Kumar, Cruess, \& Schneiderman, 1998; Scully, Kremer, Meade, Graham, \& Dudgeon, 1998; Skirka, 2000; Thayer, Newman, \& McClain, 1994; Williams \& Lord, 1997). Exercising on a treadmill produces an influx of brain serotonin (5-hydroxytryptamin: 5-HT). No indication for such findings with relaxation techniques exist (Estivill, 1995; Salmon, 1993). Previous studies on rats reveled that prolonged exercise has a protective mechanism against damage of stress and depression which is attributed to the central serotonergic and endorphin systematic reaction (Solberg, Horton, \& Turek, 1999). Rats exhibit adverse reaction when they are injected with beta-endorphin blockers (Chaouloff, 1997; Head, Kendall, Ferner, \& Eagles, 1996). A debate surrounds the question whether to contribute the positive feelings that come from the chemical neurological changes in the brain, physiological hormonal changes in the body, or cognitive-psychological ch anges in self-efficacy, perception and body image (Plante, Chizmar, \& Owen, 1999). Brain researchers have not yet determined whether it is the physical over the mental conditioning which enables humans to confront stress and depression (Steptoe, Moses, Edwards, \& Mathews, 1993).

\section{Methods}

\section{Subjects}

Subjects were adult male Wistar rats $(\mathrm{N}=18)$ weighing 200-300 g, housed in a temperature controlled room ( $23+1 \mathrm{C}$ ), and a 12/12 hrs light/dark cycle, having free access to food and water. The experiment was conducted between 10:00 and 17:00 h. 


\section{Detailed Research Plan}

Schematic presentation of the research plan:

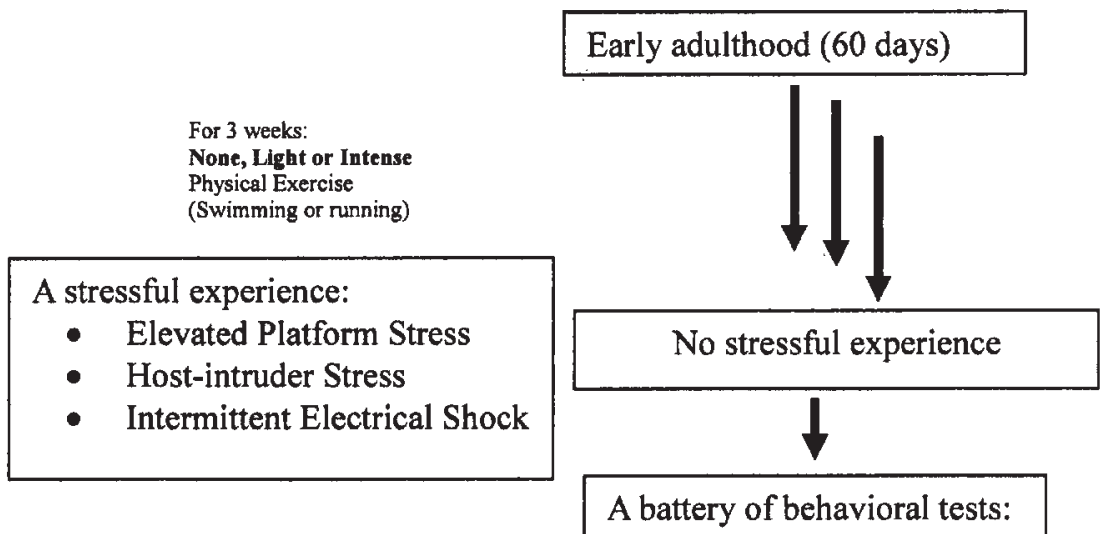

Battery A:
Exploratory behavior (Open field)
(5 min)
Anxiety level (Elevated Plus
Maze) ( 5 min)
Spatial learning (Water maze)
(1 hr)
Anxiety level (Startle reflex
response measure) (10 min)

Battery A: (5 min)

Anxiety level (Elevated Plus

Maze) (5 min)

Spatial learning (Water maze)

Anxiety level (Startle reflex

Battery B:
Exploratory behavior (Open
field) ( 5 min)
Anxiety level (Elevated Plus
Maze) ( 5 min)
Depression level (Learned
helplessness) ( 24 hrs)
Anxiety level (Startle reflex
response measure) (10 min)

Battery C:

Exploratory behavior (Open field) (5 min)

Anxiety level (Elevated Plus Maze) (5 min)

Fear learning (active avoidance) (1 hr) Anxiety level (Startle reflex response measure) $(10 \mathrm{~min})$

\section{Tissue collection for measures of neurosteroid levels}

\section{Procedures}

Rats were assigned randomly into equal six group (n = 3) as it is described in the schematic presentation of the research plan:

High intensity swimming exercise - three weeks of practice (swimming in a water maze) for a total of $40 \mathrm{~min}$. Two 20-min swimming sessions were applied with a 1-hr interval between them.

Low intensity swimming exercise - three weeks of practice (swimming in a water maze) for a total of $40 \mathrm{~min}$. Ten 4-min swimming sessions will be applied with a 30-min interval between them.

High intensity treadmill running training (HRT) three weeks of practice (running in a treadmill) for a total of $40 \mathrm{~min}$. Two 20-min swimming sessions will be applied with a 1-hr interval between them.
Low intensity treadmill running training (LRT) three weeks of practice (running in a treadmill) for a total of $40 \mathrm{~min}$. Ten 4-min swimming sessions were applied with a 30-min interval between them.

No exercise group - rats experienced stressful events, were tested for the battery of behavioral tests, but did not have exercise history.

Control group - rats were tested for the battery of behavioral tests on which the other groups were tested after the stressful events, but without experiencing the stressful events.

At the end of three weeks of intervention, rats were exposed to three stressful experiences and an hour later subjected to a battery of behavioral tests. The stressful experiences were: 
Elevated platform stress - Rats were put on an elevated platform in the middle of a water maze in a brightly lightened room. The platform was $5 \mathrm{~cm}$ above water level. The rats were held on the platform for 30 $\min$.

Host-Intruder stress - A rat was put into a cage of a territorial male rat. The territorial rat was larger than the experimental rat. The rat was left there for $30 \mathrm{~min}$. No aggressive fights were induced but rather an intensive social interaction.

Intermittent electrical shock - Rats were placed in a plastic box measuring (13"x 7.25"x 4.25") which sat on 4 piezoelectric transducers located at each of the four corners. The box was surrounded by side rail guides to limit lateral movement. Movement by the rat, including startle, caused the piezoelectric transducers to output a voltage with a linear relationship to the force applied to the floor of the cage. This system was housed inside a ventilated, lighted, sound attenuating chamber.

The voltage from the cage transducers was fed into an amplifier and then into an analogue to digital module and a PC computer at a rate of $50 \mathrm{hz} /$ channel. The signals were subsequently analyzed using analysis software programmed to quantify the peak amplitude and peak latency occurring subsequent to startle stimuli. One piezoelectric wide-dispersion horn was mounted directly over the cage. The horn delivering startle stimuli was driven by a power amplifier and a white noise generator. The signals controlling the white noise generator were also directed to a second channel of the $\mathrm{A} / \mathrm{D}$ converter input in order to document the coordination of the stimulus with the startle reactions and the signals were programmed using behavioral control software.

The rats were loosely restrained in a plexiglas tube inside the tube. A constant current shock generator provided five intermittent shocks averaging $1 \mathrm{~min}$. in 5 sec. intervals controlled by a Commodore microcomputer. In the first phase of the experiment, each subject was tested for baseline startle reactivity. Each subject was placed in the startle measurement apparatus where it was presented with a series of 150 startle-eliciting bursts of white noise (intensity $125 \mathrm{~dB}$ SPL; $0 \mathrm{msec}$ rise-fall time; duration $10 \mathrm{msec}$ ) at $6 \mathrm{sec}$ intervals. Only startle stimuli number 5-150 were considered for analysis of habituation.
In the second phase of the experiment, subjects were exposed to a three day course of traumatic shock intended to elicit a PTSD-like state. On day one, the subject received 30 tail shocks with a variable interval average $1 / \mathrm{min}$, schedule, and then returned to their home cage. On day two, after being placed in the same plexiglas cage for $8 \mathrm{~min}$, subjects received five additional tail shocks. The control group rats were placed in the shock chamber without any electric shock. In the third phase of the experiment subjects were tested for startle reactivity over time. The no training groups demonstrated non-habituation of the startle response with altered alarm responses.

\section{Measurement Instruments}

The behavioral test

The behavioral test included three batteries: a) Spatial training in the water maze (Spatial learning) (1 hour), b) Learned Helplessness in the water maze (Depression Index), and c ) Fear learning in a shuttle box (Emotional learning) (1 hour).

The batteries contained an Open Field test (exploratory behavior) (5 $\mathrm{min})$, and an Elevated Plus maze test (Anxiety Index) (5min). Immediately after being tested for their startle reflex response (Anxiety Index) $(5 \mathrm{~min})$, rats were sacrificed for tissue collection. The following brain regions were examined: prefrontal cortex, hippocampus, hypothalamus, striatum. The first three are considered to play a role in emotional and stress responses. The striatum was taken as an internal control.

The Open Field - A wooden box (90X90X38 cm), in which the floor was divided into 25 squares (17X17 $\mathrm{cm})$ by black lines. The subject was put in one of the corners and the number of squares it crossed within 5 min was taken as a measure of exploratory behavior.

The Elevated Plus maze - Four arms $(75 \times 10 \mathrm{~cm})$ organized in a + shape on a $50 \mathrm{~cm}$ high stand. Two of the arms had no walls (open arms) and the other two were protected by a $35 \mathrm{~cm}$ high opaque walls (closed arms). The subject was put in the center of the maze and the relative time spent in the closed arms was taken as an Anxiety Index (Pellow et al, 1985).

Spatial training - The water maze consisted of a circular pool of water (diameter: $1.7 \mathrm{~m}, 50 \mathrm{~cm}$ high rim). The pool was painted black. The water temperature 
was kept at $23+1^{0}$ C. For spatial training a $12 X 12 \mathrm{~cm}$ escape platform was located at one of four positions in the pool, with a top surface $2-3 \mathrm{~cm}$ below the water level. The platform was also painted black. In this way the platform, which was the only means of escape, was not visible to the swimming rat. The location of the platform was kept constant throughout training. The pool was featureless and the only obvious landmarks were those outside the water-maze in the surrounding environment. The training protocol was as follows: A rat was put to the maze with its face towards the water pool walls. Each trial the rat was randomly put at one of three possible starting points. The rat was given up to one minute to swim or until it reached the escape platform. At the end of each trial the rat was left on or brought to the escape platform for 15 seconds. Rats were given a total of 16 trials, with alternating $1 \mathrm{~min}$ and $4 \mathrm{~min}$ inter-trial intervals. At the end of the training a quadrant analysis test was performed to test for the quality of the acquisition of the spatial task. During the quadrant analysis test the escape platform was taken out. The rat was given $1 \mathrm{~min}$ to swim and its path was recorded using a video tracking system which performed an analysis of the relative distance the rat swam.

Learned Helplessness - Subjects were given a 15 min swim session on day one. On day two rats were put in the water for $2 \mathrm{~min}$ and the accumulated duration of immobility was taken as a depression index.

Fear learning - A shuttle box of two compartments and a path between them. Each trial presented a tone $(10 \mathrm{kHz}, 10 \mathrm{~s}, 75 \mathrm{~dB})$ co-terminated with an electric shock delivered through the grid floor $(1.0 \mathrm{~mA}, 5 \mathrm{~s})$ during the last $5 \mathrm{~s}$ of tone. The subject could either escape the shock or avoid it altogether by moving to the other compartment. A total of 75 trials were given with a 90 sec inter-trial interval. The number of escapes and of avoidance was calculated by a computerized system.

Startle reflex test - The startle reflex response (Partick, 1994) was measured using an automated Startle box. The high intensity exerciser rats exhibited very low startle reflex.

\section{Results}

Overall the high intensity exerciser rats performed better than the low intensity exerciser rats, who performed better than the non exerciser rats, on all tests. On the spatial training the high intensity exercisers reached the escape platform within an average of $17 \mathrm{sec}$. compared to $25 \mathrm{sec}$. of the low intensity exercisers, and $49 \mathrm{sec}$. of the non exercisers. The high intensity exercisers proved to be quick learners because they needed less number of trials to become successful (9 trials) compared to the low intensity exercisers (12 trials), and non exercisers (16 trials). On learned helplessness, the high intensity exercisers showed less immobility time (33 sec.) compared to the low intensity exercisers (39 sec.) and non exercisers (60 sec.). The high intensity exercisers found the shorter swim distance on the maze $(<1 \mathrm{~min}$.) and were able to reach the final destination within $1 \mathrm{~min}$. The other groups never made it on time. On the fear learning, the high intensity exercisers avoided the shock most of the times (97\%) out of 75 trials, compared to the low intensity exercisers (81\%), and the non exercisers (33\%). The high intensity and low intensity exerciser rats demonstrated minimal change in the startle amplitudes compared to non-habituation of the non exerciser rats. The high intensity exercisers' reaction was measured as $.18 \mathrm{~V}$ to $.30 \mathrm{~V}$ compared with the low intensity exercisers, $23 \mathrm{~V}$ to $37 \mathrm{~V}$, and the non exercisers, $.46 \mathrm{~V}$ to $.67 \mathrm{~V}$.

\section{Discussion}

In the present study, the modulation by physical exercise of the response to stressful experiences, were examined. Batteries of behavioral and biochemical measures evaluated the level of the stress response and its behavioral consequences. The three hypotheses were confirmed:

a. Physical exercise had beneficial effects on the ability of rats to cope with stressful events, similar to what was suggested in humans. The exercisers compared to non exercisers, demonstrated a moderate anxiety response, more subtle cognitive impairments and less marked depressive response when exposed to stressful events. 
b. The behavioral differences were reflected in differences in neurosteroid response. In the exerciser rats the stressful experience induced only a moderate elevation of neurosteroids concentrations in the brain, compared to the stressed, untrained rats.

c. The duration and intensity of the physical exercises influenced the magnitude of the protective effects such that more intensive physical exercise was more efficient compared to light or no training.

The susceptibility to develop affective disorders, such as anxiety, post-traumatic stress disorder, and depression are significantly influenced by the individual's life experiences, personality, and available coping mechanism. Testimonials repeatedly advocate that physical exercise has beneficial value for coping with stress and mood states disorders.

The psychophysiology of the acoustic startle reflex has been studied for decades in animals and humans. In distinction to the orienting response, which consists of a turning in the direction of a novel sound, the acoustic startle reflex is a survival mechanism of alarm that rapidly alerts and arouses an animal perceiving a loud noise. The acoustic startle mechanism in humans can be routinely measured through muscle movement, and changes in heart rate and skin conductance. The acoustic startle reflex in humans and animals has been shown to accommodate to the repeated administration of loud acoustic stimuli, such that the subject no longer startles (startle habituation; Shalev, 1992b). Similarly, the acoustic startle response accommodates to a softer pre-startle warning tone (prepulse modulation; Ornitz, 1989). PTSD is a psychiatric condition characterized by three clusters of symptoms that arise in the aftermath of a traumatic event. One cluster is composed of reexperiencing phenomena, including distressing memories of the event; the second cluster contains symptoms of avoidance of reminders of the event, and of emotional and cognitive numbing; and the third cluster includes symptoms of psychophysiological arousal (American Psychiatric Association, 1994). The no exerciser group was shown to demonstrate autonomic arousal, exaggerated startle response to acoustic stimuli, and failure to inhibit their acoustic startle reflex.

Variables such as the intensity and the duration of exercise interact with PTSD symptoms. High intensity long duration exercise suppresses the immediate physiological and psychological overwhelming stress and panic reaction. In this study, the high intensity exercisers rapidly and consistently habituated without persistently high startle reflex versus the non exerciser rates who continued to be hyper-aroused in the absence of specific cues, typified by non-habituation.

\section{References}

Anshel, M. H. (1995). Effect of chronic aerobic exercise and progressive relaxation on motor performance and affect following acute stress. Behavioral Medicine, 21, 186-196.

Atsuo, G., Kaoru, Y., Hiroshi, N., Yoshitake, T., \& Masao, O. (1995). Stress-induced elevation of ouabainlike compound in rat plasma and adrenal. Hypertension, 26, 6, 1173-1176.

Avital, A., Richter-Levin, G., Leschiner, S., Spanier, I., Veenman, L., Weizman, L., \& Gavish, M. (2001). Acute and repeated swim stress effects on peripheral benzodiazepine receptors in the rat hippocampus, adrenal, and kidney. Neuropsychopharmacology, 25(5), 669-678.

Brenner, I., Shek, P. N., Zamecnik, J., \& Shephard, R. J. (1998). Stress hormones and the immunological responses to heat and exercise. International Journal of Sports Medicine, 19(2), 130-143.

Boutcher, S. H., \& Nugent, F. W. (1993). Cardiac response of trained and untrained males to a repeated psychological stressor. Behavioral Medicine, 19, 21-27.

Brownley, K. A., West, S. G., Hinderliter, A. L., \& Light, K. C. (1996). Acute aerobic exercise reduces ambulatory blood pressure in borderline hypertensive men and women. American Journal of Hypertens, 9(3), 200-206.

Chaouloff, F. (1997). Effects of acute physical exercise on central serotonergic systems. Medical Science and Sports Exercise, 29(1), 58-62. 
Davis, M., \& Astrachan, D. I.. (1978). Conditioned fear and startle magnitude: Effects of different footshock or backshock intensities used in training. Journal of Experimental Psychology Animal Behavior Process. 4, 95-103.

Estivill, M. (1995). Therapeutic aspects of aerobic dance participation. Health Care Women International, 16, 341-350.

Fleshner, M. (2000). Exercise and neurorndocrine regulation of antibody production: Protective effect of physical activity on stress-induced suppression of the specific antibody response. Rheumatology, 57, (Suppl. 2), 6771.

Fox, K. R. (1999). The influence of physical activity on mental well being. Public Health Nutrition, 2, 411 418.

Guidotti, A., \& Costa, E. (1998). Can the antidysphoric and anxiolytic profiles of selective serotonin reuptake inhibitors be related to their ability to increase brain $3 \alpha, 5 \alpha$-tetrahydroprogesterone availability? Biological Psychiatry, 44, 865-873.

Guo, A. L., Petraglia, F., Criscuolo, M., Ficarra, G., Nappi, R. E., Palumbo, M. A., Trentini, G. P., Purdy, R. H., \& Genazzani, A. R. (1995). Evidence for a role of neurosteroids in mogulation of diurnal changes and acute stress-induced corticosterone secretion in rats. Gynecological Endocrinology, 9(1), 1-7.

Head, A., Kendall, M. J., Ferner, \& R., Eagles, C. (1996). Acute effects of beta blockade and exercise on mood and anxiety. British Journal of Sport Medicine, 30, 238-242.

Kaciuba, U. H., Porta, S., Nazar., K., Tonderska, M., Titow, S. E., Ziemba, A. W., \& Chwalbinska, M. J. (1994). Effect of mild psychological stress on physiological responses to exercise in men. Journal of Physiology and Pharmacology, 45, 429-439.

Kennedy, M. M. (1997). Effect of exercise intensity on mood in step aerobics. Journal of Sports Medicine and Physical Fitness, 37(3), 200-204.
Khisti, R. T., Chopde, C. T., \& Jain, S. P. (2000) Antidepresssant-like effect of the neurosteroid 3alphahydroxy-5alpha-pregnan-20-one in mice forced swim test. Pharmacological Biochemistry Behavior, 67(1), 137-143.

Leitner, D (1986) .Alterations in other sensory modalities accompanying stress analgesia as measured by startle reflex modification. Annals of New York Academy of Sciences 467, 82-92.

Manger , T.A. (2000). The effect of a community based aerobic exercise program on post traumatic stress disorder related symptoms and concomitant anxiety and depression. Science Engineering, 61, 1643.

McCubbib, A., Cheung, R., Montgomery, T. B., \& Bulbulian, R. (1992). Aerobic fitness and opioidergic inhilation of cardiovascular stress reactivity. Psychophysiology, 29, 687-697.

Ostrowski, K., Rohde, T., Zacho, M., Asp, B., \& Pedersen, K. (1998). Evidence that interleukin-6 is produced in human skeletal muscle during prolonged running International Journal of Sports Medicine 12, suppl. 1, S9-15.

Pederson, B. K., Bruunsgaard, H., Klokker, M., Kappel, M., MacLean, D. A., Nielsen, H. B., Rohde, T., Ullum, H., \& Zacho, M. (1997). International Journal of Sports Medicine, 18, (Suppl. 1), 2-7.

Pericic, D., Svob, D., Jazvinscak, M., \& Mirkovic, K. (2000). Anticonvulsive effect of swim stress in mice. Pharmacological Biochemistry Behavior, 66, 879-886.

Perna, F. M., Antoni, M. H., Kumar, M., Cruess, D. G., \& Schneiderman, N. (1998). Cognitive-behavioral intervention effects on mood and cortisol during exercise training. Annex Behavioral Medicine, 20(2), 92-98.

Plante, T. G., Chizmar, L., \& Owen, D. (1999). The contribution of perceived fitness to physiological and self-reported responses to laboratory stress. International Journal of Stress Management, 6, 5-19. 
Reddy, D. S., Kaur, G., \& Kulkarni, S. K. (1998). Sigma receptor mediated anti-depressant-like effects of neurosteroids in the Porsolt forced swim test. Neuroreport, 9, 3069-3073.

Robel, P., Schumacher, M., \& Baulieu, E. E. (1999). A new regulatory function in the nervous system. Totowa, NJ: Humana Press.

Robel, P., \& Baulieu, E. E. (1994). Neurosteroids biosynthesis and function. Trends in Endocrinology and Medicine, 5, 1-8.

Sallis, J. F., \& Patrick, K. (1994). Physical activity guidelines. Pediatric Exercise Science, 6, 302-314.

Salmon, S. (1993). Emotional effects of physiological exercise. San Diego, CA: Academic Press.

Scully, D., Kremer, J., Meade, M. M., Graham, R., \& Dudgeon, K. (1998). Physical exercise and psychological well being: A critical review. British Journal of Sports Medicine, 32, 111-120.

Skirka, N. (2000). The relationship of hardiness and psychological symptoms among college students. Journal of Sports Medicine and Physical Fitness, 40(1), 63-70.

Solberg, L. C., Horton, T. H., \& Turek, F. W. (1999). Circadian rhythms and depression: Effects of exercise in an animal model. American Journal of Physiology, 276(1), 151-161.

Spalding, T. W., Jeffers, L. S., Porges, S. W., \& Hatfield, B. D. (2000). Vagal and cardiac reactivity to psychological stressors in trained and untrained men. Medicine and Science in Sports and Exercise, 32, 581-591.

Steptoe, A., Moses, A., Edwards, S., \& Mathews, A. (1993). Exercise and responsibility to mental stress. International Journal of Sport Psychology, 24, 110129.

Steptoe, A., Kimbell, J., \& Basford, P. (1998). Exercise and the experience and the appraisal of daily stressors: A naturalistic study. Journal of Behavioral Medicine, 21, 363-374.
Strohle, A., Romeo, E., Herman, B., Pasini, A., Spalletta, G., Michele, F., Holsboer, F., \& Rupprecht, R. (1999). Concentration of 3 alpha-reduced neuroactive steroids and their precursors in plasma of patients with major depression and after clinical recovery. Biological Psychiatry, 45, 274-277.

Thayer, R. E., Newman, J. R., \& McClain, T. M. (1994). Self-regulation of mood. Journal of Personality and Social Psychology, 67, 910-925.

Uzunov, V., Sheline, Y., Davis, J. M., Rasmusson, A., Uzunov, D. P., Costa, A., \& Guidotti, A. (1998). Increase in the cerebrospinal fluid content of neurosteroids in patients with unipolar major depression who are receiving fluoxetine or fluoxamine. Procedures of National Academic Science, 95, 32393244.

Vallee, M., Rivera, J. D., Koob, G. F., Purdy, R. H., \& Fitzgerald, R. L. (2000). Quantification of neurosteroids in rat plasma and brain following swim stress and allopregnanolone administration using negative chemical ionization gas chromatography/mass spectrometry. Analytical Biochemistry, 287, 153-166.

Willner, P. (1984). The validity of animal models of depression. Psychopharmacology, 83, 1-16.

von Bardeleben, U., \& Holsboer, F. (1988). Human corticotropin releasing hormone. Progressive Neuropsychopharmacology Biological Psychiatry, 12 (Suppl. 1), 165-187.

Williams, P., \& Lord, S. R. (1997). Effects of group exercise on cognitive functioning and mood in older women. Austin New Zealand Journal of Public Health, 21(1), 45-52.

Willner, P. (1984). The validity of animal models of depression. Psychopharmacology, 83(1), 1-16.

\section{Correspondence:}

Author: Merav Nagel, Ph.D.

Address: Cornerstone Counseling Center

4759 Pintail CT

Salt Lake City, UT 84117

Phone: 801 262-3264

Voicemail: 801 355-3035 ext.304 